\title{
Incidence and Risk Factors for Hemorrhagic Complications after Percutaneous Renal Biopsy using a 16-Gauge Needle Followed by 6 Hours of Bed Rest
}

\section{Journal of \\ Urology \& Nephrology}

Takeshi Maehana*, Shintaro Miyamoto, Koji Ichihara, Toshiaki Tanaka and Naoya Masumori

Department of Urology, Sapporo Medical University School of Medicine, Sapporo, Japan

Address for Correspondence

Takeshi Maehana, Department of Urology, Sapporo Medical University School of Medicine, Sapporo, Japan, Tel: 81-11-611-2111; E-mail:maehana@sapmed.ac.jp

Submission: 25 April, 2017

Accepted: 30 May, 2017

Published: 12 June, 2017

Keywords: Percutaneous renal biopsy; Bed rest; Hemorrhagic complication

\begin{abstract}
Background: The procedures and management protocols for percutaneous renal biopsy (PRB) are different among institutions. Here we present the outcomes of our protocol and identify risk factors for hemorrhagic complications (HCs).
\end{abstract}

Methods: From 2007 through 2016, 175 patients received PRB using a 16-gauge biopsy needle with real-time ultrasound guidance. In a single session, three specimen cores were obtained. Post operatively, all patients were monitored with strict bed rest without sandbag compression for six hours. Adverse events were graded according to the Clavien-Dindo classification.

Results: Ninety-seven patients $(55.4 \%)$ were female and the median age was 48.0 years (IQR 37.0-64.0). The median estimated glomerular filtration rate based on serum creatinine (eGFRcreat) was $57.8 \mathrm{~mL} / \mathrm{min} / 1.73 \mathrm{~m} 2$ (IQR 28.1-87.8). The median number of glomerul assessable in each specimen was 19 (IQR 12-29). Symptomatic HCs developed in 9 patients $(5.1 \%)$. All the events occurred within 6 hours after PRB. Selective transcatheter arterial embolization and blood transfusion were required in 1 and 7 patients, respectively, but there was no grade Illb to $V$ event. In univariate analysis, predictive factors for symptomatic HCs were being female (OR 1.102, $\mathrm{p}=0.006$ ), a platelet count $<15.0 \times 104 / \mu l(O R 7.405, p=0.004)$ and eGFRcreat $<30$ $\mathrm{mL} / \mathrm{min} / 1.73 \mathrm{~m} 2$ (OR 6.300, $\mathrm{p}=0.005$ ).

Conclusion: PRB using a 16-gauge needle followed by 6 hours of strict bed rest secures sufficient samples for histological diagnosis with a low incidence of severe HCs. Furthermore, monitoring with six hours of bed rest contributes to early detection and management of severe HCs.

\section{Introduction}

Renal biopsy of native kidneys is an essential examination for diagnosis and management of patients with renal disease. The Percutaneous renal Biopsy (PRB) technique using an automated biopsy gun in combination with real-time ultrasound guidance is widely used and has become the gold standard [1]. With technological advancements, PRB has become safer, and the incidence of lifethreatening complications has decreased [2]. However, renal hemorrhage remains one of the most worrisome complications associated with PRB. Therefore, postoperative strict bed rest and careful monitoring are considered mandatory. The incidence of macroscopic hematuria and requirement for erythrocyte transfusion after PRB were reported in a systematic review to be $3.5 \%$ and $0.9 \%$, respectively [3]. In general, most severe complications become
Copyright: ๑ 2017 Maehana T, et al. This is an open access article distributed under the Creative Commons Attribution License, which permits unrestricted use, distribution, and reproduction in any medium, provided the original work is properly cited.

evident within 12 hours after the biopsy [4], but no consensus has been established for the postoperative management protocol.

According to the Japanese guideline for PRB published in 2005, the recommendations for standard care after PRB include sandbag compression at the puncture site for 2-8 hours and strict bed rest for 6-12 hours, with ambulation permitted after 18-24 hours [5]. Although longer strict bed rest potentially contributes to hemostasis after PRB, it may cause discomfort. A recently published paper reported that shortening strict bed rest from 7 to 2 hours could reduce the patient's discomfort, without an increase in the incidence of bleeding or other biopsy-related complications [6]. On the other hand, Simard-Meilleur et al. reported that $94 \%$ of complications were identified within 24 hours after PRB, and that an observation period of less than 8 hours might miss $28 \%$ of complications [7].

The size of the biopsy needle may affect the extent of renal hemorrhage. Although 14- to 18-gauge biopsy needles are usually used for RPB, the selection is dependent on the operator's preference or institutional policy. In general, a larger needle provides higherquality samples for diagnosis, but is considered to cause a higher incidence of hemorrhagic complications [3].

In 2007, we determined our standard institutional protocol for PRB, including: (1) using a 16-gauge biopsy needle, (2) monitoring with bed rest in the supine position for 6 hours after PRB, (3) no sandbag compression at the puncture site, and (4) ambulation permitted after 6 hours of bed rest if the vital signs are stable. In this study, we verified the validity and safety of our protocol and identified the risk factors for hemorrhagic complications.

\section{Materials and Methods}

\section{Inclusion criteria and PRB procedure}

This single-center retrospective study enrolled 175 consecutive patients who received PRB of a native kidney at Sapporo Medical University Hospital from April 2007 through March 2016. This series did not contain patients with a solitary kidney, and patients who underwent PRB for a renal tumor were excluded from this study. 
Citation:Maehana T, Miyamoto S, Ichihara K, Tanaka T, Masumori N. Incidence and Risk Factors for Hemorrhagic Complications after Percutaneous Renal Biopsy using a 16-Gauge Needle Followed by 6 Hours of Bed Rest. J Urol Nephrol. 2017;4(1): 5.

All patients were hospitalized and PRB was performed by urologic residents under the supervision of attending urologists. Anti platelet and anticoagulant drugs were discontinued before biopsy according to the institutional requirement and, in some cases, continuous intravenous heparinization was performed until 6 hours prior to PRB.

The PRB procedure was performed as follows. First, the patient was placed in the prone position. Then the bilateral kidneys were checked by ultrasound (Hawk 2102 EXL; B-K Medical, Denmark). A kidney with easy visualization was selected as the target of PRB and the puncture site to access the lower pole was determined. Next, the puncture site was anesthetized with using local anesthesia with $1 \%$ xylocaine after disinfection of the skin with $10 \%$ povidone iodine and a 5-mm skin incision was made there. PRB was performed using a 16-gauge automated biopsy needle (ACECUT', $16 \mathrm{G} \times 115 \mathrm{~mm}$, TSK LABORATORY, Japan). The puncture was done three times or more to obtain three sufficient cores of renal tissue. After the procedure, the puncture site was compressed manually for several minutes to control active bleeding from the incision, and then the patient was placed in the supine position.

\section{Care protocol after PRB}

Strict bed rest in the supine position without sandbag compression was required for 6 hours. Routine in dwelling bladder catheterization and administration of a hemostatic agent were not performed. Vital signs were checked at the time just after the biopsy, 1 and 6 hours later, and when patients complained any kind of symptoms. After 6 hours passed, patients without symptoms were allowed to turn over, elevate the head of the bed, and could walk for urination or defecation. The next morning (after about 18-20 hours), patients were free from restrictions except for heavy exercise and lifting of heavy weights if there were no problems with blood tests and vital signs. Routine ultrasound and computed tomography were not performed after PRB. Anti platelet and anticoagulant drugs were resumed after 2 or 3 days.

\section{Definition of complications}

Medical charts of enrolled patients were retrospectively reviewed, and all adverse events within 30 days after PRB were investigated. The severity of adverse events was graded according to the Clavien-Dindo (CD) classification [8]. In this system, any deviation from a normal postoperative course without the need for intervention was classified as Grade I. Therefore, events/symptoms that required extended bed rest for over 6 hours were defined as Grade I. In addition, any situation requiring blood transfusion, so-called hemorrhagic complications, was classified as Grade II. Grade III was for those requiring surgical, endoscopic or radiological intervention and IIIa was not under general anesthesia. In the present study, the onset time of a hemorrhagic complication was defined as the time when the patient showed the following symptoms or signs: gross hematuria, severe back pain, nausea or vomiting, hypotension, and tachycardia.

\section{Compliance with Ethical Standards}

\section{Ethical approval}

All procedures performed in studies involving human participants were in accordance with the ethical standards of the institutional research committee at which the studies were conducted (Sapporo
Medical University no. 282-44) and with the 1964 Helsinki declaration and its later amendments or comparable ethical standards.

\section{Informed consent}

Since this was an observational, but not prospective, intervention study, the Ethics Committee provided a waiver of written informed consent. We announced the commencement of this study on our website (http://web.sapmed.ac.jp/uro/) with the proviso that the patients who participated in this study could withdraw later.

\section{Statistical Analysis}

The patients' characteristics are presented using the median and interquartile range (IQR) for continuous variables. The estimated glomerular filtration rate based on serum creatinine (eGFRcreat) was calculated as follows: eGFRcreat $\left(\mathrm{mL} / \mathrm{min} / 1.73 \mathrm{~m}^{2}\right)=194 \times$ serum creatinine $(\mathrm{sCr})-1.094 \times$ age- 0.287 (in female: $\times$ 0.739) [9]. Comparisons between two groups were performed using Fisher's exact test and the chi-square test. $\mathrm{P}$ values of less than 0.05 were considered statistically significant. All statistical analyses were performed with EZR statistical software (Saitama Medical Center, Jichi Medical University) [10], which is a graphical user interface for $\mathrm{R}$ (The R Foundation for Statistical Computing, version 2.13.0). More precisely, it is a modified version of $\mathrm{R}$ Commander (version 1.6-3) that includes the statistical functions frequently used in biostatistics.

\section{Results}

The characteristics of the patients are shown in Table1. The median age of the 174 patients was 48.0 years (IQR 37.0-64.0), and $55.4 \%$ were female. The median eGFRcreat was $57.8 \mathrm{~mL} / \mathrm{min} / 1.73$ $\mathrm{m}^{2}$ (IQR 28.1-87.8). The numbers of punctures in a single session of PRB to obtain three sufficient cores for evaluation were $3,4,5$ and 6 or more in $46.6 \%, 31.0 \%, 10.3 \%$ and $12.0 \%$, respectively (median 4 , range 3-12). A median of 19 glomeruli (IQR 12-29) were present and

Table 1: Characteristics of patients who received percutaneous renal biopsy.

\begin{tabular}{|c|c|}
\hline Parameter & Value \\
\hline Male/Female, $n(\%)$ & $78(44.6) / 97(55.4)$ \\
\hline Median age, years (IQR) & $48.0(37.0-64.0)$ \\
\hline Median BMI, kg/m² (IQR) & $22.0(19.8-24.7)$ \\
\hline \multicolumn{2}{|l|}{ Comorbidity, n (\%) } \\
\hline Hypertension & $54(30.9)$ \\
\hline Diabetes mellitus & $14(8.0)$ \\
\hline Ischemic heart disease & $10(5.7)$ \\
\hline Dyslipidemia & $26(14.9)$ \\
\hline Anticoagulation or antiplatelet therapy, $\mathrm{n}(\%)$ & $52(29.7)$ \\
\hline Systemic heparinization, $\mathrm{n}(\%)$ & $25(14.3)$ \\
\hline \multicolumn{2}{|l|}{ Pre-biopsy laboratory parameters, median (IQR) } \\
\hline Hemoglobin, g/dl & $11.9(9.9-13.5)$ \\
\hline Platelets, $\times 10^{4} / \mu 1$ & $24.7(19.5-29.7)$ \\
\hline Serum creatinine, $\mathrm{mg} / \mathrm{dl}$ & $0.90(0.70-1.86)$ \\
\hline eGFRcreat, $\mathrm{m} / / \mathrm{min} / 1.73 \mathrm{~m}^{2}$ & $57.8(28.1-87.7)$ \\
\hline \multicolumn{2}{|l|}{ Puncture side, $\mathrm{n}(\%)$} \\
\hline Right/Left & $115(65.7) / 60(34.3)$ \\
\hline \multicolumn{2}{|l|}{ Number of punctures, $n(\%)$} \\
\hline $3 / \geq 4$ & $82(46.9) / 93(53.1)$ \\
\hline
\end{tabular}

BMI: Body Mass Index; eGFRcreat: estimated Glomerular Filtration Rate creatinine 
Citation:Maehana T, Miyamoto S, Ichihara K, Tanaka T, Masumori N. Incidence and Risk Factors for Hemorrhagic Complications after Percutaneous Renal Biopsy using a 16-Gauge Needle Followed by 6 Hours of Bed Rest. J Urol Nephrol. 2017;4(1): 5.

assessable in each core specimen.

There were no grade IIIb or more complications associated with PRB according to the CD classification in this cohort. In addition, there was no complication other than hemorrhagic complications. Hemorrhagic complications requiring extended bed rest over 6 hours were observed in 9patients (5.1\%). Six patients (3.4\%) had grade II (requiring transfusion) and 1 patient $(0.6 \%)$ had grade IIIa (treated with percutaneous selective transcatheter arterial embolization). In univariate analysis, female gender, a platelet count $<15.0 \times 10^{4} / \mu$ and eGFRcreat $<30 \mathrm{~mL} / \mathrm{min} / 1.73 \mathrm{~m}^{2}$ were significantly associated with

Table 2: Univariate analysis of risk factors for hemorrhagic complications after PRB.

\begin{tabular}{|c|c|c|c|c|c|}
\hline Variables & $\begin{array}{c}\text { With } \\
\text { complication } \\
(n=9)\end{array}$ & $\begin{array}{c}\text { Without } \\
\text { complication } \\
(n=166)\end{array}$ & $\begin{array}{c}P \\
\text { value }\end{array}$ & $95 \% \mathrm{Cl}$ & $\begin{array}{l}\text { Odds } \\
\text { ratio }\end{array}$ \\
\hline Female gender, $\mathrm{n}(\%)$ & $9(100)$ & $88(53.0)$ & 0.006 & $\begin{array}{c}1.034- \\
1.175\end{array}$ & 1.102 \\
\hline Age $\geq 60$ years, $n(\%)$ & $5(55.6)$ & $52(31.3)$ & 0.470 & & \\
\hline $\mathrm{BMI} \geq 25 \mathrm{~kg} / \mathrm{m}^{2}, \mathrm{n}(\%)$ & $1(11.1)$ & $40(24.1)$ & 0.687 & & \\
\hline Hypertension, n (\%) & $2(22.2)$ & $73(44.0)$ & 0.080 & & \\
\hline Diabetes mellitus, $\mathrm{n}(\%)$ & $0(0.0)$ & $19(11.4)$ & 0.600 & & \\
\hline $\begin{array}{c}\text { Ischemic heart disease, } \\
\mathrm{n}(\%)\end{array}$ & $0(0.0)$ & $10(6.0)$ & 1.000 & & \\
\hline Dyslipidemia, n (\%) & $0(0.0)$ & $26(15.7)$ & 0.359 & & \\
\hline $\begin{array}{l}\text { Anticoagulation or } \\
\text { antiplatelet therapy, } \\
n(\%)\end{array}$ & $2(22.2)$ & $51(30.7)$ & 0.457 & & \\
\hline $\begin{array}{c}\text { Systemic } \\
\text { heparinization, } \mathrm{n}(\%)\end{array}$ & $2(22.2)$ & $23(13.9)$ & 0.613 & & \\
\hline $\begin{array}{c}\text { Hemoglobin }<11.0 \mathrm{~g} / \\
\text { dl, }(\%)\end{array}$ & $5(55.6)$ & $59(35.6)$ & 0.290 & & \\
\hline $\begin{array}{c}\text { Platelets }<15.0 \times 10^{4} / \\
\mu \mathrm{l},(\%)\end{array}$ & $3(33.3)$ & $11(6.6)$ & 0.004 & $\begin{array}{l}1.549- \\
32.048\end{array}$ & 7.045 \\
\hline $\begin{array}{c}\text { eGFR }<30 \mathrm{ml} / \mathrm{min} / 1.73 \\
\mathrm{~m}^{2}, \mathrm{n}(\%)\end{array}$ & $6(66.7)$ & $40(24,1)$ & 0.005 & $\begin{array}{l}1.506- \\
26.347\end{array}$ & 6.300 \\
\hline $\begin{array}{c}\text { Number of punctures } \geq \\
4, \mathrm{n}(\%)\end{array}$ & $6(66.7)$ & $87(52.4)$ & 0.731 & & \\
\hline
\end{tabular}

BMI: Body Mass Index; eGFRcreat: estimated Glomerular Filtration Rate creatinine

Table 3: Details of 9 patients with hemorrhagic complications.

\begin{tabular}{|c|c|c|c|c|c|c|c|c|c|c|c|c|c|}
\hline No. & Age & Sex & BMI & $\begin{array}{c}\text { Anticoagulation } \\
\text { therapy }\end{array}$ & $\begin{array}{l}\text { Pre } \\
\mathrm{Hb}\end{array}$ & $\begin{array}{l}\text { Pre } \\
\text { PIt }\end{array}$ & $\begin{array}{c}\text { Pre } \\
\text { eGFRcreat }\end{array}$ & $\begin{array}{l}\text { Number of } \\
\text { punctures }\end{array}$ & $\begin{array}{c}\text { Time of onset } \\
\text { (hrs after } \\
\text { biopsy) }\end{array}$ & $\begin{array}{l}\text { Symptoms at } \\
\text { onset time }\end{array}$ & Treatment & $\begin{array}{c}\text { CD } \\
\text { classification }\end{array}$ & $\begin{array}{l}\text { Total rest } \\
\text { time (hrs) }\end{array}$ \\
\hline 1 & 53 & Female & 22.7 & Warfarin & 12.1 & 15.2 & 58.6 & 6 & 2 & Back pain & - & I & 24 \\
\hline 2 & 20 & Female & 18.6 & Warfarin & 14.2 & 25.9 & 129.5 & 4 & 1 & Tachycardia & - & I & 48 \\
\hline 3 & 67 & Female & 22.4 & - & 9.4 & 3.6 & 25.6 & 4 & 2 & Hypotension & Transfusion & II & 24 \\
\hline 4 & 41 & Female & 27.7 & - & 10.9 & 29.7 & 11.1 & 8 & 2 & Hypotension & Transfusion & II & 24 \\
\hline 5 & 71 & Female & 19.8 & - & 8.9 & 29.1 & 16.2 & 4 & 1 & $\begin{array}{c}\text { Gross } \\
\text { hematuria with } \\
\text { clot retention }\end{array}$ & $\begin{array}{c}\text { Transfusion, } \\
\text { Continuous } \\
\text { bladder } \\
\text { irrigation }\end{array}$ & II & 48 \\
\hline 6 & 64 & Female & 22.0 & - & 9.5 & 29.7 & 6.1 & 4 & 2 & Vomiting & Transfusion & II & 24 \\
\hline 7 & 60 & Female & 17.0 & - & 12.0 & 21.9 & 26.5 & 3 & 5 & Hypotension & Transfusion & II & 12 \\
\hline 8 & 70 & Female & 19.5 & - & 12.2 & 9.8 & 53.3 & 3 & 2 & $\begin{array}{c}\text { Back pain } \\
\text { Hypotension } \\
\text { Tachycardia }\end{array}$ & $\begin{array}{c}\text { Transfusion, } \\
\text { Selective } \\
\text { TAE }\end{array}$ & IIla & 24 \\
\hline 9 & 22 & Female & 21.2 & - & 9.1 & 11.7 & 18.7 & 4 & 5 & $\begin{array}{l}\text { Back pain, } \\
\text { Vomiting }\end{array}$ & Transfusion & II & 24 \\
\hline
\end{tabular}

BMI: Body Mass Index; Hb: Hemoglobin, Plt: Platelets; eGFRcreat: estimated Glomerular Filtration Rate creatinine; hrs: hours; TAE: Transcatheter Arterial Embolization; CD: Clavien-Dindo the development of hemorrhagic complications (Table 2). Details of the patients with hemorrhagic complications are shown in Table 3. In all patients these events were diagnosed within 6 hours after PRB. The total times of bed rest after PRB were 12, 24 and 48 hours for 1, 6, and 2 patients, respectively.

\section{Discussion}

The purposes of strict bed rest after PRB are: (1) to avoid expansion of renal hemorrhage and (2) to monitor symptoms and signs such as gross hematuria, flank pain, hypotension and tachycardia, which are suggestive of active renal hemorrhage. In those with unstable hemorrhage, further careful monitoring with strict bed rest is needed and blood transfusion and intervention must be considered. Conventional standard management after RPB includes 24 hours of bed rest after the biopsy in both the inpatient and outpatient settings [4]. In Japan, PRB is commonly performed in the inpatient setting. The standard post-PRB care recommendation includes compression of the biopsy site with sandbags or a bandage for 2-8 hours, and strict bed rest for 6-12 hours. Moreover, patients remain in bed for an additional 12 hours and are finally allowed to stand and walk 18-24 hours after RPB [5]. However, controversy has emerged regarding the optimal duration of strict bed rest and monitoring. Several studies showed that outpatient observation for 6-8 hours was safe enough $[7,11,12]$. On the other hand, prolonged bed rest after the procedure is not always significantly safe [13]. In 2009, Ishikawa et al. reported that no significant differences were observed between 2 and 7 hours of strict rest with respect to severe bleeding complications or progression of anemia after PRB [6]. In their series, only 2 of 94 patients $(2.1 \%)$ who were put on strict rest in the decubitus position for 2 hours after PRB needed transfusion or hemostatic intervention. Therefore, strict rest for a long duration after PRB may not be required to avoid expansion of hemorrhage. In our series, the incidence of transfusion or intervention was $4.0 \%$, which is comparable with other reports $[7,14]$. Moreover, all of the events were detected within 6 hours after the procedure. These results suggest that strict bed rest with monitoring for 6 hours after PRB is 
Citation:Maehana T, Miyamoto S, Ichihara K, Tanaka T, Masumori N. Incidence and Risk Factors for Hemorrhagic Complications after Percutaneous Renal Biopsy using a 16-Gauge Needle Followed by 6 Hours of Bed Rest. J Urol Nephrol. 2017;4(1): 5.

reasonable and that patients can be allowed to be out of bed after 6 hours of bed rest safely if they have stable vital signs with no specific symptoms.

Frequent, strict monitoring of vital signs and renal hemorrhage is employed in some institutions. Whittier et al. checked vital signs every 15 minutes for 2 hours, every hour for 4 hours, every 2 hours for 6 hours, and then every 4 hours up to 24 hours postPRB [14]. Moreover, single voided urine was visually inspected for gross hematuria, and hemoglobin levels were checked at 5-8, 10-13 and 18-20 hours post-RPB. Routine ultrasound examination was performed at 1 hour after PRB. In contrast, our study adopted a simple protocol. Vital signs were routinely checked only at 1 and 6 hours after PRB unless the patient complained of specific symptoms. Routine ultrasound examination was not done and a blood test was only performed the next morning. Even so, we did not overlook clinically significant hemorrhagic complications, suggesting that our simple protocol was safe and sufficient for post-PRB management Furthermore, discomfort associated with frequent blood drawing and examination should be avoided as much as possible.

In recent review articles, hemorrhagic complications associated with PRB of native kidneys were reported to occur in $3.0-8.0 \%$ of cases $[3,15]$. In addition, the transfusion rates were said to be $0.9-6.3 \%$ $[7,16]$. However, the exact incidence and severity of the complications was unclear because the evaluation methods for complications associated with PRB were different among the reports. To evaluate the incidence and severity of postoperative complications, the CD classification has generally been used $[17,18]$. This classification may also be applicable for evaluation of post-PRB complications.

Age, female gender, elevated blood pressure, a low baseline hemoglobin level and platelet count, elevated $\mathrm{sCr}$ (the same as low eGFRcreat), and prolonged bleeding time were reported to be risk factors for hemorrhagic complications after PRB [1,15,19-21]. In a meta-analysis by Corapi et al. age, female gender, hypertension, increased $\mathrm{sCr}$ and the use of a 14-gauge needle were independently associated with hemorrhagic complications [3]. Although multivariate analysis could not be performed due to the small number of events in the present study, our results are in accord with other reports. We should thus anticipate hemorrhagic complications needing transfusion and interventions after PRB, especially in cases with such risk factors, and provide sufficient information to the patients before PRB. Furthermore, we should also consider open or laparoscopic biopsy that can secure certain hemostasis during the procedure in such cases.

The transfusion rate in our series was comparable with those in other reports where a 16-gauge needle was mainly used $[7,14,16]$. However, the rate might be slightly higher than that in a contemporary report, in which an 18-gauge needle was used [6]. The decision for transfusion is dependent on physicians and the patients conditions are not always uniform. Transfusion might have been over treatment in some cases of our series. On the other hand, the size of the needle might affect the incidence of hemorrhagic complications [3]. We obtained a sufficient number of glomeruli in each specimen. The quality of samples might be quite high as compared with other reports $[7,22]$. Renal biopsy is an essential examination for the diagnosis and management of patients with renal disease. Therefore, highquality samples should be secured as long as the procedure can be done safely. Simard-Meilleur et al. reported that an 18-gauge needle provided a significantly fewer glomeluri in a specimen, although there was a trend toward a lower rate of symptomatic hematomas [7]. Considering the above and our findings together, usage of 16-gauge needle may be beneficial.

There were some limitations in this study. First, the number of events was small due to the small sample size. Therefore, independent risk factors for hemorrhagic complications could not be determined. Second, this study did not have a control group, so the superiority or non-inferiority of our PRB management protocol could not be tested. Third, the retrospective single center nature of this study limits its generalizability. Therefore, a multicenter prospective randomized controlled study with larger populations may be required to confirm the validity of the protocol.

\section{Conclusion}

In conclusion, the incidence of complications associated with PRB using a 16-gauge needle was 5.1\%, and female gender, a low platelet count and low eGFRcreat were significant risk factors in our series. Hemorrhagic complications of grade II and III were observed in $3.4 \%$ and $0.6 \%$ of the cases, respectively, all of which were detected within 6 hours post-RPB. All biopsy specimens contained a sufficient number of glomeruli. These results suggest that our 6-hour strictbed-rest protocol after 16-gauge needle PRB is safe and reasonable.

\section{Conflicts of interest statement and funding}

Koji Ichihara has received Grants from Kyowa Hakko Kirin Co., Ltd. (KHKS20160517012).

\section{References}

1. Eiro M, Katoh T, Watanabe T (2005) Risk factors for bleeding complications in percutaneous renal biopsy. Clin Exp Nephrol 9: 40-45.

2. Kim D, Kim H, Shin G, Ku S, Ma K, et al. (1998) A randomized, prospective, comparative study of manual and automated renal biopsies. Am J Kidney Dis 32: $426-431$

3. Corapi KM, Chen JL, Balk EM, Gordon CE (2012) Bleeding complications of native kidney biopsy: a systematic review and meta-analysis. Am J Kidney Dis 60: 62-73.

4. Marwah DS, Korbet SM (1996) Timing of complications in percutaneous renal biopsy: what is the optimal period of observation? Am J Kidney Dis 28: $47-52$.

5. Nishi S (2005) Prescription of bed-rest after renal biopsy. Nihon Jinzo Gakkai Shi 47: 491-496.

6. Ishikawa E, Nomura S, Obe T, Katayama K, Oosugi K, et al. (2009) How long is strict bed rest necessary after renal biopsy? Clin Exp Nephrol 13 594-597.

7. Simard-Meilleur MC, Troyanov S, Roy L, Dalaire E, Brachemi S (2014) Risk factors and timing of native kidney biopsy complications. Nephron Extra 4 42-49.

8. Dindo D, Demartines N, Clavien PA (2004) Classification of surgical complications: a new proposal with evaluation in a cohort of 6336 patients and results of a survey. Ann Surg 240: 205-213.

9. Matsuo S, Imai E, Horio M, Yasuda Y, Tomita K, et al. (2009) Revised equations for estimated GFR from serum creatinine in Japan. Am J Kidney Dis 53: $982-992$ 
Citation:Maehana T, Miyamoto S, Ichihara K, Tanaka T, Masumori N. Incidence and Risk Factors for Hemorrhagic Complications after Percutaneous Renal Biopsy using a 16-Gauge Needle Followed by 6 Hours of Bed Rest. J Urol Nephrol. 2017;4(1): 5.

ISSN: 2380-0585

10. Kanda $Y(2012)$ Free statistical software: EZR (Easy R) on R commander.

11. Lin WC, Yang Y, Wen YK, Chang CC (2006) Outpatient versus inpatient renal biopsy: a retrospective study. Clin Nephrol 66: 17-24.

12. Maya ID, Allon M (2009) Percutaneous renal biopsy: outpatient observation without hospitalization is safe. Semin Dial 22: 458-461.

13. Allen C, Glasziou P, Del Mar C (1999) Bed rest: a potentially harmful treatment needing more careful evaluation. Lancet 354:1229-1233.

14. Whittier WL, Sayeed K, Korbet SM (2016) Clinical factors influencing the decision to transfuse after percutaneous native kidney biopsy. Clin Kidney J 9: 102-107.

15. Tondel C, Vikse BE, Bostad L, Svarstad E (2012) Safety and complications of percutaneous kidney biopsies in 715 children and 8573 adults in Norway 1988-2010. Clin J Am Soc Nephrol 7: 1591-1597.

16. Stratta $\mathrm{P}$, Canavese $\mathrm{C}$, Marengo M, Mesiano P, Besso L, et al. (2007) Risk management of renal biopsy: 1387 cases over 30 years in a single centre. Eur J Clin Invest 37: 954-963.
17. Losa A, Gadda GM, Lazzeri M, Lughezzani G, Cardone G, et al. (2013) Complications and quality of life after template-assisted transperineal prostate biopsy in patients eligible for focal therapy. Urology 81: 1291 1296.

18. Clavien PA, Barkun J, de Oliveira ML, Vauthey JN, Dindo D, et al. (2009) The Clavien-Dindo classification of surgical complications: five-year experience. Ann Surg 250: 187-196.

19. Korbet SM, Volpini KC, Whittier WL (2014) Percutaneous renal biopsy of native kidneys: a single-center experience of 1,055 biopsies. Am J Nephrol 39: 153-162.

20. Manno C, Strippoli GF, Arnesano L, Bonifati C, Campobasso N, et al. (2004) Predictors of bleeding complications in percutaneous ultrasound-guided renal biopsy. Kidney Int 66: 1570-1577.

21. Whittier WL, Korbet SM (2004) Timing of complications in percutaneous renal biopsy. J Am Soc Nephrol 15: 142-147.

22. Torres Muñoz A, Valdez-Ortiz R, González-Parra C, Espinoza-Dávila E, Morales-Buenrostro LE, et al. (2011) Percutaneous renal biopsy of native kidneys: efficiency, safety and risk factors associated with major complications. Arch Med Sci 823-831.

\section{Acknowledgements}

The authors thank Mr. Kim Barrymore for English proofreading of this manuscript. 